\title{
Análisis de portafolio por sectores mediante el uso de algoritmos genéticos: caso aplicado a la Bolsa Mexicana de Valores
}

Fecha de recepción: 21.01.2014

Fecha de aceptación: 20.05.2014

Martha del Pilar
Rodríguez García
Universidad Autónoma de
Nuevo León
marthadelpilar2000@yahoo.com

Klender Aimer Cortez

Alejandro

Universidad Autónoma de

Nuevo León

klender@yahoo.com

\section{Alma Berenice Méndez \\ Sáenz \\ Universidad Autónoma de \\ Nuevo León \\ beremendez@hotmail.com}

Héctor Horacio Garza

Sánchez.

Universidad Autónoma de

Nuevo León

hfacpya@hotmail.com

\section{Resumen}

El tipo de sector, el tamaño de la empresa, el número de trabajadores, etc. son variables que se consideran de control en una gran cantidad de publicaciones. En este trabajo consideramos estudiar la variable sector - más que como una variable de control - como una variable determinante del desempeño financiero (Baird et al. 2012) y del riesgo (Artikis y Nifora, 2011). Así, se analiza seis sectores de la economía mexicana divididos de acuerdo con la Bolsa Mexicana de Valores en Industrial, Productos de consumo básico, Materiales, Productos de consumo no básico, Telecomunicaciones y Servicios financieros. La muestra se compone de 30 empresas mexicanas dentro del periodo de 2007-2012. Para medir el desempeño del portafolio se utilizan dos indicadores clásicos: (1) Alfa de Jensen y (2) Ratio de Sharpe; se utiliza una métrica condicional que mide el número de veces que el rendimiento del portafolio supera el rendimiento promedio del mercado. El objetivo es encontrar un portafolio que maximice estos parámetros y comparar los resultados entre los diferentes sectores bajo estudio. Debido a un problema de programación no lineal, se utilizan algoritmos genéticos para obtener el portafolio óptimo que maximice estas métricas. Los resultados muestran un mejor desempeño financiero ajustado a riesgo en el sector de Materiales y Servicios financieros y un desempeño más bajo en sectores como el Industrial y el de Telecomunicaciones.

Palabras clave: desempeño sectorial, portafolio, Alfa de Jensen, Ratio de Sharpe, algoritmos genéticos 


\title{
Sectorial portfolio analysis with genetic algorithms: case applied to the Mexican Stock Exchange
}

\begin{abstract}
The type of industry, size of company, number of employees, etc. are variables that are considered as control variables in a large number of articles. In this research we consider the sector variable as a determinant of financial performance (Baird et al. 2012) and the risk (Artikis and Nifora, 2011) rather than as a control variable. This paper analyzes six sectors of the Mexican economy divided according to the Mexican Stock Exchange: industrial, basic consumer products, materials, non basic consumer products, telecommunications and financial services. The sample consists of Mexican companies, that is, 30 companies in the 2007-2012 period. To measure portfolio performance two classic indicators are used: (1) Jensen alpha and (2) Sharpe ratio, and also conditional metrics are used that measures the number of times the portfolio return exceeds the market average. The goal is to find a portfolio that maximizes these parameters and compare the results between the different sectors under study. Due to a nonlinear programming problem, genetic algorithms are used to obtain the optimal portfolio that maximizes these metrics. The results show a better risk-adjusted financial performance in the field of materials and financial services and a lower performance in such sectors as the industrial and telecommunications ones.
\end{abstract}

Key words: sectorial performance, portfolio, Alpha Jensen, Sharpe Ratio, genetic algorithms

\section{Introducción}

Los problemas en los mercados financieros internacionales durante 2007 tuvieron un menor impacto sobre las economías emergentes que episodios previos de turbulencia financiera. Lo anterior se explica en parte por la mejora en las condiciones económicas fundamentales de este grupo de países en años recientes, así como por la trayectoria favorable de sus términos de intercambio. A pesar de ello, los sectores productivos en México fueron afectados (Banxico, 2008).

Nuestro estudio comienza en el 2007, cuando la actividad económica presentó un menor dinamismo comparado con años anteriores debido a que el Producto Interno Bruto (PIB) registró un crecimiento real anual de 3.3\%, que se compara con $4.8 \%$ en 2006. Esta desaceleración se originó, principalmente, por un menor crecimiento de la demanda externa, aunque también el gasto interno atenuó su ritmo de expansión. La inversión, en particular la realizada por el sector privado, también redujo su tasa de crecimiento. El incremento del PIB de 3.3\% en 2007 se 
derivó de aumentos de $4.4 \%$ del sector servicios, de $1.4 \%$ del industrial y de $2 \%$ del agropecuario. En relación con 2008, el PIB creció apenas $1.3 \%$ derivado de bajos crecimientos sectoriales, esto es, $2.1 \%$ y $3.2 \%$ en los servicios y en el sector agropecuario, respectivamente, y de una contracción de $0.7 \%$ en los niveles de actividad del sector industrial.

Según el reporte anual de Banxico (2009), la caída de 0.7\% anual que registró la producción del sector secundario (industrial) en 2008 se vio influida por las reducciones observadas en la minería, las manufacturas y la construcción de $2.3 \%$, $0.4 \%$ y $0.6 \%$, respectivamente. Por su parte, la electricidad presentó un aumento de 2.2\%. En el caso de la minería, ésta acumuló dos años con variaciones anuales negativas. Asimismo, la disminución que registró la producción manufacturera en 2008 estuvo influida por el menor dinamismo de las exportaciones efectuadas por dicho sector, resultado de la recesión en Estados Unidos y en las economías de otros socios comerciales, así como por la desaceleración de la demanda interna por sus productos.

Por último, el sector más dinámico dentro de las actividades terciarias, con un aumento de $8 \%$ en el año, fue el rubro de información en medios masivos, apoyado en los renglones de telefonía celular y de comunicaciones satelitales. Otros sectores con un comportamiento favorable fueron los correspondientes a la actividad de dirección de corporativos y empresas y el relacionado con los servicios inmobiliarios y de alquiler, los cuales presentaron incrementos de $4.2 \%$ y $3.2 \%$, respectivamente. En lo que respecta al sector de transportes, correos y almacenamiento, éste tuvo una tasa positiva de sólo $0.8 \%$ en el 2008 , destacando las alzas que observaron sus componentes de autotransporte de carga y de servicios de mensajería. El comercio, a su vez, presentó un avance anual en este periodo de $2.8 \%$. En este último sector destacó la desaceleración que mostraron las ventas minoristas en el mercado nacional.

Las características del desempeño sectorial en México son fuertemente influidas por el impacto de las inversiones extranjeras y existen realmente sesgos en el desarrollo sectorial. El crecimiento de la inversión en el nivel mundial durante la presente década ha estado concentrado en el sector servicios. Al contrario de lo que ocurre en manufacturas - que de representar cerca del 30\% de la inversión total realizada en el exterior por los países de la OCDE, en la segunda mitad de los años noventa pasó a menos de $20 \%$ en el periodo 2000-2004-, los servicios han incrementado significativamente su importancia (BBVA, 2007). Es importante 
resaltar que dentro del sector servicios los mayores flujos se han orientado al sector financiero. De un total de 50 mil millones de dólares de inversión orientados a servicios, el 70\% (35 mil) correspondió al renglón de servicios financieros; esto es, la concentración de la inversión en pocas actividades ha sido una característica de la inversión extranjera: del total registrado desde la firma del Tratado de Libre Comercio de América del Norte hasta 2006, $90 \%$ se ubica en manufacturas, servicios financieros, comercio y transporte y comunicaciones. Para el caso específico de las manufacturas, tan sólo las divisiones de maquinaria y equipo, alimentos, bebidas y tabaco, y química, hule y plástico, representaron más del $80 \%$ del total.

Recientemente en el año 2012, la economía mexicana registró una trayectoria positiva, cuyo ritmo de crecimiento condujo a que la actividad continuara convergiendo de manera ordenada hacia niveles congruentes con el potencial productivo del país. Hacia la segunda mitad de 2012 dicho entorno ocasionó una pérdida de dinamismo de la economía nacional. Este menor ritmo de crecimiento se derivó inicialmente de menores tasas de expansión de las exportaciones manufactureras $\mathrm{y}$, posteriormente, de la transmisión de este choque a algunos componentes de la demanda interna.

La inversión mostró una notoria desaceleración en la segunda mitad de 2012 debido, principalmente, al débil desempeño de la inversión en construcción, especialmente en lo relacionado con la construcción de vivienda, aun cuando el gasto en inversión en maquinaria y equipo también presentó una pérdida de dinamismo. Esta desaceleración fue particularmente notoria en el sector industrial como reflejo, fundamentalmente, de una disminución en el ritmo de crecimiento del sector manufacturero y del de la construcción (Banxico, 2012).

Por su parte, durante 2012 el sector servicios presentó una tendencia positiva, si bien su tasa de expansión en el segundo semestre de ese año fue ligeramente menor a la que se observó en promedio durante la primera mitad. Este comportamiento se derivó, principalmente, de la transmisión de la desaceleración de la demanda externa a los servicios más asociados a ésta, los cuales incluso mostraron una contracción en el tercer trimestre del año. En particular, la disminución en los servicios más asociados a la demanda externa fue reflejo, a su vez, de la caída en los servicios del sector comercio en dicho periodo. Por su parte, el sector de servicios de transporte, correos y almacenamiento presentó variaciones positivas en el segundo semestre de 2012, si bien de menor magnitud a las exhibidas en la primera parte del año. En contraste, algunos servicios asociados a la demanda interna continuaron 
presentando un ritmo de crecimiento relativamente elevado, como es el caso de los servicios inmobiliarios y de alquiler, los de alojamiento temporal y preparación de alimentos y bebidas, los servicios educativos y los de salud (Banxico, 2012).

En lo que corresponde al sector industrial, donde se encuentran las principales constructoras de México, el año actual ha sido de franca caída (BBVA, 2013). El modelo bajo el cual crecieron estas empresas fue exitoso en su momento, pero mostró sus debilidades y signos de desgaste hacia finales de la década pasada. Hubo señales importantes en este sentido que no fueron adecuadamente valoradas. Sin embargo, debe decirse que en algunos aspectos relevantes para medir la operación de estas empresas la información era incompleta o poco transparente. También es cierto que el propio modelo que habían adoptado les restaba flexibilidad. El crecimiento que el sector mostró por arriba de la economía no se mantendría en el corto plazo. Sin embargo, la desaceleración ha sido mayor a lo esperado e incluso el sector está cayendo. Este resultado se debe a que sus dos principales componentes, la edificación y la obra civil, han presentado resultados negativos en el cuarto trimestre de 2012 y en el primero de 2013.

Desde la perspectiva de las empresas y sus propietarios, la obtención de beneficios es el principal objetivo e impulsor de la actividad de la empresa. El beneficio puede ser considerado como el resultado de todos los esfuerzos realizados para el mantenimiento de su actividad, mientras que el desempeño financiero de las empresas muestra la eficacia para transformar los ingresos en renta para que, posteriormente, pueda ser distribuido a los accionistas. El desempeño financiero es el resultado de varios factores tanto internos como externos; dentro de los internos se encuentran la productividad de la compañía, eficiencia, eficacia, desempeño de los administradores, gobierno corporativo e innovación, en tanto que dentro de los externos tenemos condiciones macroeconómicas, monetarias y sectoriales (Gruian, 2011).

El factor externo del sector dentro del desempeño financiero será el analizado en este estudio para lo cual Baležentis et al. (2012) mencionan que la comparación entre sectores económicos sigue siendo un área subdesarrollada, por lo que en la mayoría de las investigaciones sólo se han estudiado eficiencia energética, comercio internacional o sectores específicos. Dentro de la revisión de literatura se encontraron algunas investigaciones que han analizado dicha relación para la Unión Europea (UE) (Comisión Europea, 2009; Gruian, 2011), en Dhaka, Bangladesh (Mahmud, 2011), Milán, Italia (Artikis y Nifora, 2011) y para el caso mexicano Saavedra y Saavedra (2012). 
El objetivo de este trabajo es examinar el desempeño financiero de los seis sectores representativos de la Bolsa Mexicana de Valores (BMV) e identificar si existe una diferencia significativa en sus respectivos rendimientos y riesgos. Se aplicaron diversas metodologías para el análisis de portafolios como rendimiento y varianza del portafolio, Ratio de Sharpe, Alfa de Jensen y algoritmos genéticos. Dentro de este artículo se presenta en primer lugar una revisión de literatura sobre el desempeño de los diferentes sectores; en segundo lugar, se muestra el marco teórico referente a la Teoría de Portafolio Moderna; posteriormente, explicamos la metodología de algoritmos genéticos, así como también la muestra y periodo seleccionado; por último, se exponen los resultados obtenidos y las conclusiones.

\section{Revisión de la literatura}

El sector, el tamaño de las empresas, la tecnología y el riesgo son variables que tienen efectos en el desempeño financiero de las empresas (Baird et al. 2012). Existen algunos estudios tipo panel que utilizan como variable de control el tipo de sector para determinar el nivel de desempeño financiero (Artikis y Nifora 2011). En el siguiente apartado exponemos algunos hallazgos acerca del efecto del sector en el desempeño financiero, medido en términos de riesgo y desempeño financiero.

En Gruian (2011) se realiza un estudio en donde se seleccionaron ocho países de la Unión Europea (UE) divididos en trece sectores no financieros para el periodo de 2009. El estudio muestra que los sectores más rentables son el sector minero, servicios profesionales, actividades científicas y técnológicas y servicios públicos; mientras que los peores son el transporte, la agricultura, el alojamiento y restaurantes. Además, encuentran que las industrias más vulnerables en tiempos de crisis económica y financiera son transporte, la agricultura, el alojamiento y la alimentación, servicios y manufactura.

En otro estudio, Mahmud (2011) emplea una muestra representativa de $126 \mathrm{em}-$ presas listadas en la Bolsa de Valores de Dhaka, las cuales se dividieron en diez sectores. Los hallazgos muestran que el sector de la ropa de vestir es uno de los más rentables, seguido por el sector bancario y de seguros; sin embargo, estos últimos dos sectores presentan un menor grado de riesgo comparado con el sector de vestir, por lo que la evidencia demuestra que el sector bancario es el mejor sector para invertir. Por otra parte, el sector bancario es influido por las variables macro económicas. 
La Comisión Europea tiene estudios en donde se analiza la competitividad y la rentabilidad de empresas de la UE bajo la perspectiva sectorial (Comisión Europea, 2009). El periodo considerado es 1995-2005. En la UE los mejores son el sector servicios, los servicios públicos, la minería y otros sectores basados en las materias primas y algunas industrias manufactureras, en especial la fabricación de coque, petróleo refinado y combustible nuclear y la fabricación de pasta de papel, papel y productos de papel que agregan valor a la materia prima en las industrias de la minería y la silvicultura. En el otro extremo de la jerarquía podemos encontrar la agricultura y el transporte aéreo, las cuales tuvieron un margen neto negativo en el periodo considerado. Otras industrias de bajo rendimiento son el sector manufacturero y comercio al mayoreo y al menudeo y construcción.

Artikis y Nifora (2011) muestran, además del efecto en el desempeño del sector, el efecto del nivel de endeudamiento en los rendimientos de sus acciones de acuerdo con la clasificación sectorial de la empresa, ya que el sector es considerado como un factor de riesgo importante. En el estudio aplicado a la Bolsa de Valores de Milán utilizan empresas no financieras durante el periodo de 2000 a 2010. Se hicieron divisiones sectoriales con base en la clasificación industrial de Bloomberg (tecnología, petróleo y gas, material básico, bienes de consumo, servicios a los consumidores, cuidado personal, industria, telecomunicaciones y servicios públicos). La industria del cuidado personal es el segundo sector que tiene un mayor nivel de apalancamiento después de servicios públicos y parece tener el mayor rendimiento promedio para todo el periodo investigado. Por otra parte, servicios a los consumidores, que presenta un bajo nivel de apalancamiento junto con tecnología, tiene el segundo lugar en menor rentabilidad promedio.

Para el caso de México encontramos literatura relacionada con el tema en el estudio de Saavedra y Saavedra (2012), en el cual se selecciona una muestra de 31 empresas durante el periodo de 2001 al 2008 considerando los sectores que pertenecen al sector alimentos, bebidas y tabaco, comunicaciones, comercio, construcción, extractivo y vivienda. Mide el desempeño financiero a través del Valor Económico Agregado (VEA) y con el precio de la acción. En su estudio obtiene que en todos los sectores se creó valor excepto en los sectores construcción y vivienda, los que además son más sensibles a los efectos de las crisis económicas. En cambio, si el desempeño financiero se mide con el precio de la acción, aquél es creciente en todos los sectores hasta el año 2006; sin embargo, en el sector vivienda para el año 2007 la tendencia es decreciente. El crecimiento del precio de mercado para todos los sectores resultó negativa para el periodo 2007-2008; los sectores que resultaron 
con mayor decrecimiento son construcción $66 \%$, vivienda 53\%, extractivo $51 \%$ y alimentos, bebidas y tabaco $28 \%$, con lo que se muestra el efecto negativo que tuvo la crisis financiera de 2008.

En este estudio, mediante la teoría de portafolio, se pretende encontrar algún patrón de diferencias en rendimiento y riesgo, considerando esta última variable como valor agregado, pues no ha sido utilizado en ningún estudio de desempeño financiero sectorial. Por ello, se desea probar si el sector tiene efectos significativos en rendimiento de mercado y riesgo.

\section{Marco teórico}

El uso de la Teoría Moderna del Portafolio (TMP), compuesta por la Teoría de Selección de Portafolio establecida por Markowitz en 1952 y por las contribuciones a la teoría de formación de precios de los activos financieros introducida por Sharpe en 1964 (Mangram, 2013), se refiere a un marco de inversión para la selección y construcción de portafolio basado en la maximización de la rentabilidad esperada del portafolio y la minimización del riesgo (Fabozzi et al., 2002). El teorema fundamental de media-varianza de un portafolio propuesto por Markowitz en 1952 supone maximizar un rendimiento esperado manteniendo una varianza dada o minimizando una varianza con un rendimiento de portafolio dado. Si todas las combinaciones posibles de las inversiones se presentan en un gráfico de riesgos y rendimientos, los puntos definen una región en este espacio limitado por una frontera eficiente (FE) (Markowitz, 1952; Merton, 1972; Chamberlain, 1983; Owen y Rabinovitch, 1983).

La principal consideración de la TMP es que la selección de una acción no es determinada sólo con sus características únicas entre el rendimiento-riesgo de la acción, sino que el inversionista debe considerar también la covarianza de la acción con un grupo de acciones de un índice objetivo. Considerando estas covarianzas entre las acciones es posible construir portafolios que tengan el mismo rendimiento esperado con un menor riesgo que un portafolio que ignore estos covarianzas entre las acciones.

En este trabajo se propone el uso de la TMP como método para encontrar la combinación de acciones de empresas que maximice la relación riesgo-rendimiento de un índice compuesto de acciones y, por otra parte, determinar si la razón riesgo-rendimiento difiere entre sectores. La fórmula para el rendimiento del portafolio se expresa como sigue: 


$$
\bar{R}_{p}=\frac{\sum_{i=1}^{n} x_{i} \bar{R}_{i}}{\sum_{i=1}^{n} x_{i}}
$$

donde:

$x_{i}=$ Peso de cada uno de los activos individuales que conforman el portafolio.

$\bar{R}_{i}=$ Rendimientos esperados de los activos individuales.

$\bar{R}_{p}=$ Rendimiento promedio del portafolio.

Mientras que la varianza del portafolio se expresa con la siguiente ecuación:

$$
\sigma_{p}^{2}=\sum_{j=1}^{N} \sum_{k=1}^{N} x_{j} x_{k} \sigma_{j, k}
$$

donde:

$x_{j}, x_{k}=$ Representan los pesos dentro del portafolio de los activos $j, k$.

$N=$ número de empresas que forman parte del portafolio

$=$ Covarianzas $j, k$.

$\sigma_{p}^{2}=$ Varianza de portafolio.

$\sigma_{j, k}=\sigma_{j}^{2}=\sigma_{k}^{2}, \quad$ si $\quad j=k$

Para determinar el desempeño del portafolio entre los diferentes sectores es necesario calcular ciertas métricas. Uno de los primeros estudios que evaluó el desempeño de un portafolio fue el realizado por Cowles (1933).

Los primeros estudios realizados emplearon una variedad de técnicas para la evaluación de portafolios como el ratio de Sharpe, el cual utiliza el riesgo total (Sharpe, 1966), y el alfa de Jensen, que utiliza la beta como la medida correcta del riesgo (Jensen, 1968 y 1969). Estas dos métricas se describirán a continuación.

El alfa de Jensen (1968 y 1969) es una medida del grado en el cual el rendimiento promedio del portafolio responsable excede el rendimiento del portafolio del mercado como a continuación se expresa en la siguiente ecuación: 
donde:

$$
\alpha=R_{P}-\left[R_{F}+\beta\left(R_{m}-R_{f}\right)\right]
$$

$\alpha=$ alfa de Jensen

$R_{P}=$ Rendimiento esperado del portafolio.

$R_{f}=$ Rendimiento libre de riesgo

$R_{m}=$ Rendimiento de mercado del portafolio

El ratio de Sharpe considera el riesgo y rendimiento de índices. Este ratio puede ser utilizado para expresar qué tan bien el rendimiento de un activo compensa el riesgo asumido por un inversionista (Sharpe, 1994). Se define como:

$$
S=\frac{R_{P}-R_{f}}{\sigma_{P}}
$$

donde:

$R_{P}=$ rendimiento esperado del portafolio

$R_{f}=$ rendimiento de la tasa libre de riesgo

= riesgo esperado medido con la desviación estándar del portafolio

Si la $R_{f}$ está libre de riesgo entonces la varianza de los rendimientos es cero; esto es, la desviación estándar del exceso es la misma que la desviación estándar de los rendimientos del activo (Scholz, 2007). Cada una de las acciones tendrá diferentes perfiles de riesgo-rendimiento o distintas razones de Sharpe.

Otra métrica que puede resultar interesante al momento de evaluar un desempeño de un portafolio es el número de veces que el rendimiento de éste supera el rendimiento promedio del mercado, el cual denotamos como el Total de Ganancias (TG) y que puede ser calculado mediante la siguiente expresión condicional:

donde:

$$
T G=\frac{1}{N} \sum_{t=1}^{n} v_{t}
$$

n: es el número de observaciones.

$$
v_{t}=\left\{\begin{array}{l}
1 \text { si }\left(R_{P}-R_{M}\right)_{t}>0 \\
0 \text { si }\left(R_{P}-R_{M}\right)_{t} \leq 0
\end{array}\right.
$$


Podemos seleccionar un portafolio de inversión buscando la combinación de acciones que maximice alguna o todas las métricas anteriores. Para ello resulta conveniente buscar una técnica que ayude a lograr este objetivo.

\section{Metodología}

Los algoritmos genéticos (AG) desarrollados por Holland (1975) y Michalewicz (1996) permiten analizar desde una perspectiva distinta a la programación lineal. Los AG son algoritmos de optimización estocásticos basados en el mecanismo de selección natural y genética (Krishnasamy, 2011; Sefiane y Benbouziane, 2012). $\mathrm{Su}$ principal ventaja es que no es necesario especificar todos los detalles de la formulación de un problema con antelación (Roudier, 2007), además de ser adecuados en la solución de problemas complejos (Krishnasamy, 2011). Existen diversos artículos que han utilizado los AG para resolver el problema de selección de portafolios como Armone et al. (1993), Venugopal et al. (2004), Kyong et al. (2005), Lin y Liu (2008), Yu et al. (2009) y Yu et al. (2012), Sefiane y Benbouziane (2012), entre otros.

Arnone et al. (1993) utilizaron AG para la optimización de un portafolio sin restricciones con un riesgo a la baja. Venugopal et al. (2004) muestran un modelo de AG que considera capital y deuda que permite el cambio de deuda por capital en fase alcista y viceversa. Kyong et al. (2005) también emplearon AG para fundamentar la optimización de un fondo. Lin y Liu (2008) propusieron los AG para problemas de selección de portafolios con lotes mínimos de transacción. Para resolver el problema de selección y análisis de portafolios de atributos múltiples Yu et al. (2009) y Yu et al. (2012) aplicaron los AG.

En los AG se genera aleatoriamente una población inicial, la cual contiene un número constante de cromosomas. En un problema de optimización de un portafolio, cada cromosoma representa el peso de la acción individual del portafolio para llegar a una posible solución óptima. Mediante el uso de cruce, mutación y la selección de valores que cumplen con las restricciones, la población convergerá a una que contiene cromosomas únicos con una buena forma física. Cuanto mayor es el valor de la aptitud, el valor de la función objetivo será mejor. Los pasos básicos mencionados por Chang et al. (2009) para crear un AG son los siguientes 
1. Iniciar una población generada aleatoriamente.

2. Evaluación de la aptitud del individuo en la población.

3. Aplicar selección elitista: llevar a los mejores individuos para la próxima generación de la reproducción mediante cruces y mutaciones.

4. Reemplazar la población actual por la nueva población.

5. Si las restricciones se cumple y se ha llegado a un óptimo entonces el proceso se detiene, de lo contrario ir al paso 2.

Aunque el proceso de optimización podría ser teóricamente cierto, en los AG existen algunos problemas en la práctica como mencionan Tarek et al. (2006), principalmente por el supuesto que asumió Holland (1975) sobre el tamaño infinito de la población. Sin embargo, en la práctica se utiliza una población finita y la interacción de los "genes" es muy pequeña, ya que influye en la habilidad de generar las muestras del algoritmo genético y afectando su desempeño. Por consiguiente, si se utiliza un método de búsqueda local puede mejorar los resultados de la optimización porque al introducir nuevos "genes" puede ayudar a combatir el problema causado por la acumulación de errores estocásticos derivadas de una población finita (Asoh y Mühlenbein, 1994).

Otro problema de los AG es la selección inapropiada de los parámetros de control como el tamaño de la población, la tasa de cruce y la tasa de mutación, pues estos parámetros podrían generar soluciones diferentes en su proceso de optimización (Deb, 1997). La selección del parámetro correcto nos puede llevar a un consumo mayor de tiempo como señalan Tarek et al. (1996). Debido a estas limitantes los AG se pueden integrar con otros métodos de búsqueda para mejorar su desempeño. Talbi (2002) señala que los AG ofrecen mejoras significativas, principalmente en la velocidad de la búsqueda, cuando se combinan con métodos que utilicen una búsqueda local.

Los métodos que usan este tipo de búsqueda utilizan el conocimiento local de los individuos para propagar sus características en la siguiente generación y mejorar la solución óptima, por eso la búsqueda local es considerada como un proceso de aprendizaje (Tarek et al., 1996). Existen principalmente dos enfoques que han sido empleados: 1) aprendizaje lamarckiano ${ }^{1}$ que se basa en características no hereditarias adquiridas que se obtienen del aprendizaje y 2) aprendizaje baldwiniano que,

\footnotetext{
${ }^{1}$ La aplicación del aprendizaje lamarckiano ha sido efectiva en resolver el problema del TSP (Traveling Salesman Problem).
} 
a diferencia del anterior, no permite que las características aprendidas se pasen a sus descendientes. Existen varias perspectivas sobre el desempeño al utilizar AG puros o híbridos, una revisión sobre estas investigaciones se pueden consultar en Tarek et al. (1996).

\section{Muestra}

Para el caso de México se obtuvo información completa de 28 de las 35 empresas que componen el Índice de Precios y Cotizaciones (IPC). La muestra empleada se agrupa a partir de siete sectores obtenidos de la Bolsa Mexicana de Valores (BMV). Los sectores son productos de consumo básico, materiales, telecomunicaciones, industrial, productos de consumo no básico y servicios financieros. Con base en los sectores de la BMV se construyeron seis portafolios uno para cada sector dentro del IPC.

La muestra está compuesta por aquellas emisoras que han sido consideradas dentro del IPC durante el 2007 al 2012. Aunque es un periodo de crisis propiciada por Estados Unidos, en el 2008 los canales externos de transmisión fueron diferentes en comparación a crisis pasadas. Como resultado de la significativa mejora en los balances externos durante el auge precedente, y de la serie de estímulos y paquetes de rescate de los países industrializados, los canales de transmisión financieros fueron más débiles y más breves en su duración que en las crisis anteriores. Los países disfrutaron de algún espacio para las políticas macroeconómicas anticíclicas (Ocampo, 2011). Además, la investigación se beneficia por contar con un estudio reciente donde el efecto de la crisis se presente en todos y cada uno de los sectores. En el cuadro 1 se presenta la muestra de empresas por sector.

\section{Cuadro 1}

\section{Empresas de la muestra}

\begin{tabular}{c|c|c|c|c|c}
\hline Industrial & $\begin{array}{c}\text { Productos } \\
\text { de Consumo } \\
\text { Básico }\end{array}$ & Materiales & $\begin{array}{c}\text { Productos de } \\
\text { Consumo No } \\
\text { Básico }\end{array}$ & $\begin{array}{c}\text { Telecomuni- } \\
\text { caciones }\end{array}$ & $\begin{array}{c}\text { Servicios } \\
\text { Financieros }\end{array}$ \\
\hline ALFA & AC & CEMEX & ALSEA & AXTEL & BANORTE \\
\hline ARA & BIMBO & GMEXICO & ELEKTRA & TLEVISA & BBVA \\
\hline ASUR & COMERCI & PEÑOLES & LIVERPOOL & AMX & CITIGROUP \\
\hline GAP & FEMSA & MEXCHEM & & & VALUEGF \\
\hline GEO & GMODELO & & & & \\
\hline HOMEX & GRUMA & & & & \\
\hline ICA & KIMBER & & & & \\
\hline URBI & SORIANA & & & &
\end{tabular}

Fuente: Infosel (2012) 
Para efectos de este estudio hemos consultado la base de datos de Infosel ${ }^{2}$ para obtener las series de los valores mensuales de los precios para cada empresa, así como también del IPC. Para la tasa libre de riesgo utilizamos los CETES a 28 días considerando los rendimientos anuales mínimos y máximos de cada mes obtenidos a través del Banco de México³.

\section{Frontera eficiente}

Para elaborar la frontera eficiente (FE) se utilizó los datos mensuales de precios para cada uno de los seis sectores. Con estos precios se calcularon los rendimientos y la desviación estándar de cada portafolio. En la construcción de las FE para cada uno de los seis sectores seleccionados se determinó un polígono de combinaciones factibles; es decir, fuera de esta área no es posible conseguir una combinación riesgo-rendimiento con las empresas de la muestra. Para calcular la frontera superior del polígono resolvimos el siguiente problema de programación lineal como sigue:

donde:

$$
\begin{gathered}
\operatorname{Min} \quad \mathrm{S}_{p}^{2} \\
\text { s.a: } \bar{R}_{p}=r ; \quad 0 \leq x \leq 1 ; \quad \sum_{i=1}^{T} x_{i}
\end{gathered}
$$

$\mathrm{r}=\mathrm{r}_{\mathrm{j}}^{\min }, \ldots ., \mathrm{r}_{\mathrm{k}}^{\max }$

$\mathrm{r}_{\mathrm{j}}^{\mathrm{min}}=$ valor de la acción con mínimo rendimiento

$\mathrm{r}_{\mathrm{k}}{ }^{\max }=$ valor de la acción con máximo rendimiento.

Por otro lado, la frontera interna del polígono se obtuvo uniendo los puntos donde se coloca toda la inversión en una acción en particular del portafolio.

Una vez construidas las FE de los portafolios de inversión por sector estimamos las tres métricas para evaluar portafolios señaladas en las ecuaciones (3), (4) y (5). El objetivo es encontrar la combinación de pesos de las acciones dentro del portafolio que maximicen estas tres métricas. Como tenemos tres objetivos, uno por cada métrica, se requiere la creación de una función que combine los tres objetivos en uno solo. Para ello, se utilizó un Ratio Ponderado (RP) que representa la media ponderada de las tres métricas. Lo anterior nos lleva a un problema de programación no

${ }^{2} \mathrm{http}: / /$ mxplusinvertia.com

${ }^{3}$ http://www. banxico.org.mx 
lineal, ya que la función que deseamos maximizar incluye métricas calculadas a partir de una regresión lineal (alfa de Jensen) así como la métrica condicionante de la ecuación (5). Además se consideró como restricción principal que se tuviera en la medida de lo posible resultados con valores estadísticamente significativos de la métrica del alfa de Jensen. Se agregó también la restricción de que el peso mínimo de cada empresa fuera más del $1 \%$ con la finalidad de que todas las empresas fueran incluidas. Lo anterior, dificulta el uso de la programación lineal; por ello optamos por los algoritmos genéticos propuestos por Holland (1975), los cuales ha sido utilizado para evaluar portafolios de inversión. El problema de optimización queda definido como sigue:

$$
\begin{gathered}
\text { Max: } \frac{R_{p}-\left[R_{F}+\beta\left(R_{m}-R_{f}\right)\right]}{3}+\frac{R_{P}-R_{f}}{3 \sigma_{P}}+\frac{\sum_{t=1}^{N} v_{t}}{3 N} \\
\text { s.a } \\
p-\operatorname{valor}() \leq 0.1 \\
\sum_{i=1}^{N} x_{i}=1 \\
0.01 \leq x_{i} \leq 1
\end{gathered}
$$

\section{Software empleado y parámetros de control}

En relación con el software utilizado se optó por utilizar Evolver versión 6; tiene la ventaja de aplicar algoritmos genéticos en conjunto con el generador de optimización matemática OptQuest para buscar soluciones óptimas a un problema específico. Este generador utiliza optimización matemática metaheurística y componentes de una red neuronal para guiar la búsqueda de las mejores soluciones para problemas de decisión y planificación de todo tipo. Los métodos de OptQuest ${ }^{4}$ integran procedimientos metaheurísticos como la búsqueda tabú ${ }^{5}$, las redes neuronales artificiales y la búsqueda dispersa, en un solo método compuesto. Dado que

${ }^{4}$ OptQuest es un producto registrado de OptTek Systems.

${ }^{5} \mathrm{La}$ búsqueda tabú aumenta el rendimiento del método de búsqueda local mediante el uso de estructuras de memoria, ya que una vez que una potencial solución es determinada se la marca como "tabú" de modo que el algoritmo no vuelva a visitar esa posible solución, véase Glover \& Laguna (1997). 
el método de búsqueda dispersa ${ }^{6}$ se basa en realizar combinaciones y aplicar métodos de búsqueda local, Martí y Laguna (2002) señalan que se puede considerar incluido en los llamados algoritmos lamarckianos.

Sobre el proceso de optimización, se utilizaron como valores iniciales de cada portafolio la misma ponderación para todas las empresas que lo integran. También cabe señalar que como parámetro de paro o tiempo de ejecución se consideró un umbral de $0.00001 \%$ de cambio en los pesos; es decir, si la celda objetivo no se modifica en un $0.00001 \%$ entonces el algoritmo se detiene, lo anterior con la finalidad de reducir el tiempo de optimización. En relación con la técnica de optimización, o sea, el algoritmo genético u OptQuest, se utilizaron ambos.

En el caso del algoritmo genético se consideraron los parámetros establecidos por defecto en Evolver. El tamaño de población indica cuántos organismos (o grupos completos de variables) deben almacenarse en memoria en cada momento. En Palisase (2013) se señala que hay mucho debate e investigación sobre el tamaño óptimo de una población para diferentes problemas, pero se recomienda usar entre 30 y 100; en nuestro caso utilizamos el valor de 80 . La tasa de cruce refleja la probabilidad de que futuros escenarios u "organismos" contengan una mezcla de información de la generación previa de organismos originales; para este parámetro se usó un valor de 0.50 , es decir, un organismo descendiente contiene aproximadamente el $50 \%$ de sus valores de variable de un "padre" y el resto de los valores del otro "padre". La tasa de mutación refleja la probabilidad de que futuros escenarios contengan algunos valores aleatorios; para este trabajo se consideró un valor de 0.1 .

\section{Resultados}

En México, para cualquier nivel dado de riesgo esperado, se tiene un rendimiento esperado mayor en el sector de materiales como se puede observar en la figura 1; estas conclusiones son muy similares a los estudios de Gruian (2011) y Comisión Europea (2009), que mencionan que éste es uno de los sectores con mayor rentabilidad aunque no mencionan la variable riesgo.

${ }^{6}$ La búsqueda dispersa (scatter search) es un método evolutivo utilizado para la resolución de problemas de optimización. Aunque presenta similitudes con los algoritmos genéticos, difiere de éstos en principios fundamentales, tales como el uso de estrategias sistemáticas en lugar de aleatorias, véase Glover et al. (2000). 
Es importante destacar que el sector de servicios financieros también tiene un rendimiento alto, seguido del sector de materiales. Por su parte, el sector telecomunicaciones tiene una frontera eficiente con menor rendimiento, que también es considerado con pobre desempeño financiero en el trabajo de Gruian (2011), quien menciona que este sector - al igual que otros, como agricultura, alojamiento y restaurantes - además de presentar menor rentabilidad es de los más vulnerables ante las crisis financieras.

\section{Figura 1}

Frontera eficiente por sectores 2008-2012

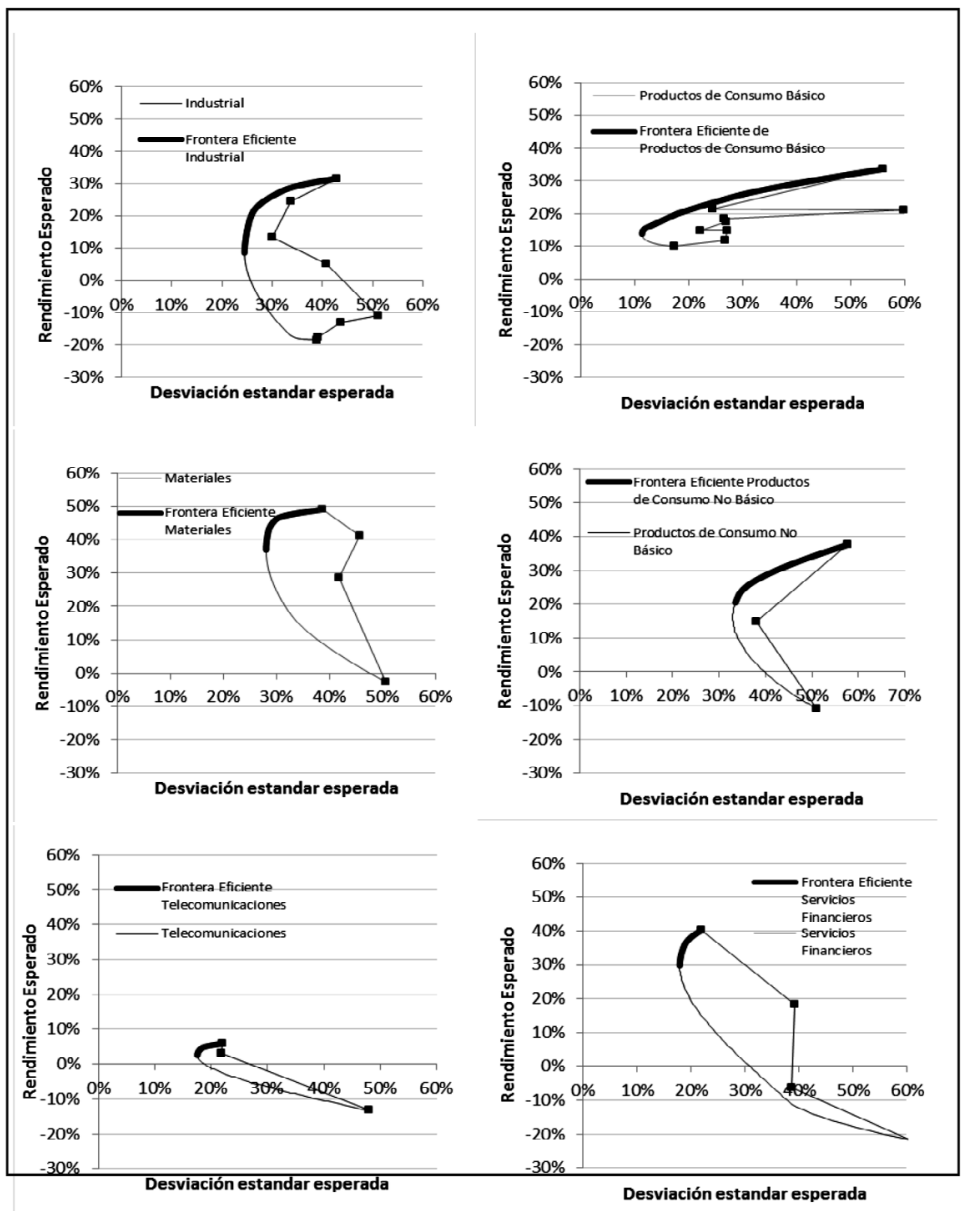

Fuente: Elaboración propia usando Evolver. 
Con el fin de maximizar las tres métricas de desempeño de las ecuaciones (3), (4) y (5) decidimos optimizar el valor de una ratio ponderada considerando un $33 \%$ de peso para cada una de estas métricas. Para ello utilizamos algoritmos genéticos y el generador OptQuest. Como se puede observar en el cuadro 2, la diferencia entre ambas técnicas fue principalmente en el tiempo en que se logró el valor óptimo, ya que el generador OptQuest alcanzó en menor lapso de tiempo optimizar la ratio ponderada. Sin embargo, el valor óptimo fue muy similar con ambos métodos.

\section{Cuadro 2}

\section{Resultados de la optimización}

\begin{tabular}{c|c|c|c|c|cc}
\hline \multirow{2}{*}{ Sector } & \multicolumn{3}{|c|}{ Algoritmo genético } & \multicolumn{3}{c}{ OptQuest } \\
\cline { 2 - 7 } & $\begin{array}{c}\text { Número } \\
\text { de } \\
\text { pruebas }\end{array}$ & $\begin{array}{c}\text { Tiempo } \\
\text { (hh:mm:ss) }\end{array}$ & Valor óptimo & $\begin{array}{c}\text { Número de } \\
\text { pruebas }\end{array}$ & $\begin{array}{c}\text { Tiempo } \\
\text { (hh:mm:ss) }\end{array}$ & $\begin{array}{c}\text { Valor } \\
\text { optimo }\end{array}$ \\
\hline Industrial & 4859 & $00: 01: 08$ & $46.6461 \%$ & 1700 & $00: 00: 31$ & $46.6480 \%$ \\
\hline $\begin{array}{c}\text { Productos } \\
\text { de Consumo } \\
\text { Básico }\end{array}$ & 7810 & $00: 02: 28$ & $63.4154 \%$ & 1400 & $00: 00: 34$ & $62.768 \%$ \\
\hline Materiales & 2737 & $00: 01: 05$ & $66.7554 \%$ & 952 & $00: 00: 46$ & $66.7604 \%$ \\
\hline $\begin{array}{c}\text { Productos de } \\
\text { Consumo No } \\
\text { Básico }\end{array}$ & 2434 & $00: 00: 39$ & $52.889 \%$ & 891 & $00: 00: 24$ & $52.885 \%$ \\
\hline $\begin{array}{c}\text { Telecomunica- } \\
\text { ciones }\end{array}$ & 2631 & $00: 00: 53$ & $6.1250 \%$ & 923 & $00: 00: 24$ & $6.1258 \%$ \\
\hline $\begin{array}{c}\text { Servicios } \\
\text { Financieros }\end{array}$ & 2019 & $00: 00: 44$ & $82.3519 \%$ & 513 & $00: 00: 14$ & $82.3531 \%$ \\
\hline
\end{tabular}

Debido a que el valor óptimo con ambas metodologías fue muy semejante y al extensivo uso de los AG en el campo financiero (Vallée y Yildizoglu, 2003), en particular en el pronóstico de rendimientos y optimización de portafolios (Pereira, 2000), optamos por considerar los AG para interpretar nuestros resultados, ya que el objetivo de este trabajo es analizar el desempeño financiero de los sectores de las empresas que integran el Índice de Precios y Cotizaciones mediante el uso de AG.

La ratio ponderada muestra que definitivamente el sector con mejor desempeño mediante la optimización con algoritmos genéticos es el relacionado con los servicios financieros; los mismos resultados obtiene Mahmud (2011). Le sigue, en orden de importancia, materiales y bienes de consumo básico. Los resultados con más bajo desempeño resultaron nuevamente ser los que se presentan en el sector de telecomunicaciones. 
Para no determinar la relación de rendimiento-riesgo a través de la FE, sino determinar el desempeño de los portafolios para cada uno de los sectores, analizamos las métricas de desempeño de cada portafolio (empresas del mismo sector) que se obtuvieron del proceso de optimización con algoritmos genéticos. El cuadro 3 muestra los resultados. En primer lugar, si consideramos el porcentaje de veces que el portafolio del sector le gana al mercado, los sectores de productos de consumo básico, materiales y servicios financieros superan el desempeño del índice de mercado en un $86.67 \%$; mientras que el sector de telecomunicaciones fue el sector con menor porcentaje de desempeño comparado con el índice de referencia.

En segundo lugar, considerando la introducción del riesgo sistemático medido por Beta y el modelo de alfa de Jensen, los resultados cambian para el sector de bienes de consumo básico, ya que se colocó entre los sectores con menor desempeño medido en términos de riesgo y rendimiento. Sin embargo, los resultados se mantienen para los sectores de servicios financieros y materiales con los valores más altos en términos de alfa de Jensen, mientras que el sector de telecomunicaciones tiene un valor negativo, lo cual indica que este sector no presenta rendimientos en exceso o anormales.

En tercer lugar, si consideramos el ratio de Sharpe que mide qué tan bien el rendimiento del portafolio por sector supera al riesgo, los sectores de servicios financieros y productos de consumo básico obtienen los mayores valores, esto es, 1.1588 y 0.8573 respectivamente, seguido por el sector de materiales con un valor de 0.7520 .

Tabla 3

Métricas de desempeño como resultado de la optimización con algoritmos genéticos

\begin{tabular}{c|c|c|c|c|c|c}
\hline $\begin{array}{c}\text { Métrica de } \\
\text { desempeño }\end{array}$ & Industrial & $\begin{array}{c}\text { Productos } \\
\text { de consumo } \\
\text { básico }\end{array}$ & Materiales & $\begin{array}{c}\text { Productos de } \\
\text { consumo no } \\
\text { básico }\end{array}$ & $\begin{array}{c}\text { Telecomunica- } \\
\text { ciones }\end{array}$ & $\begin{array}{c}\text { Servicios } \\
\text { financieros }\end{array}$ \\
\hline $\begin{array}{c}\% \text { sobre el } \\
\text { Indice de } \\
\text { Mercado= }\end{array}$ & $68.33 \%$ & $86.67 \%$ & $86.67 \%$ & $63.33 \%$ & $28.33 \%$ & $86.67 \%$ \\
\hline $\begin{array}{c}\text { Alfa de } \\
\text { Jensen= }\end{array}$ & $27.19 \%$ & $15.99 \%$ & $38.42 \%$ & $24.95 \%$ & $-4.68 \%$ & $44.51 \%$ \\
\hline t-student & $(6.6463)$ & $(0.1599)$ & $(7.0229)$ & $(5.2267)$ & $(-3.4707)$ & $(9.1550)$ \\
\hline p-valor & $(0.0000)$ & $(0.0000)$ & $(0.0000)$ & $(0.0000)$ & $(0.0010)$ & $(0.0000)$ \\
\hline $\begin{array}{c}\text { Ratio de } \\
\text { Sharpe= }\end{array}$ & 0.4443 & 0.8573 & 0.7520 & 0.7038 & -0.0529 & 1.1588 \\
\hline
\end{tabular}

Fuente: Elaboración propia utilizando Evolver 6. 
En la figura 2 se presentan los resultados a partir de la relación riesgo-rendimiento, se puede observar que a pesar de tener niveles parecidos de rendimiento los sectores servicios financieros, materiales e industrial muestran riesgos muy diferentes; de ellos el sector industrial es el más riesgoso. Por otra parte, los sectores de productos de consumo básico y no básico muestran relaciones de riesgo-rendimiento muy similares. Finalmente, se observa que el sector de telecomunicaciones presenta el nivel más bajo de rendimiento y de riesgo.

\section{Figura 2}

\section{Rendimiento vs riesgo en portafolios optimizados}

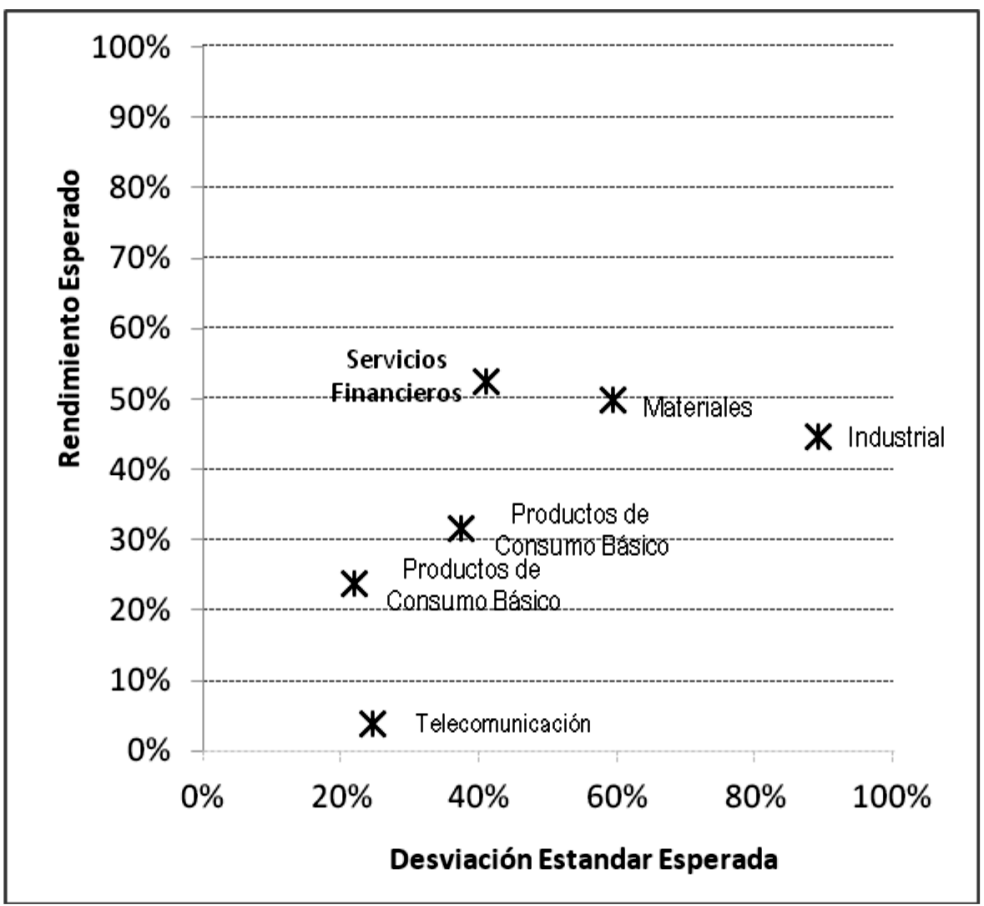

Fuente: Elaboración propia usando Evolver 6.

\section{Conclusiones}

Las empresas se encuentran continuamente buscando estrategias que maximicen su relación riesgo y rendimiento a través de la toma de decisiones basadas en va- 
riables fundamentales como la estructura de costos, de endeudamiento y sus proyecciones de ventas. Pero lamentablemente el panorama externo al que se encuentran expuestas, esto es, cambios en las políticas monetarias, fiscales nacionales e internacionales, por mencionar algunas, afectan de manera drástica su gestión. Los cambios económicos tienes diferentes efectos si lo comparamos por sectores. Es por ello que en este estudio analizamos si el sector es una variable determinante para la relación riesgo-rendimiento; además, si los resultados que encontramos son similares o diferentes de los trabajos revisados en la literatura. Los hallazgos de este artículo nos llevan a pensar que diferentes oportunidades de inversión por sectores contribuirán a diferentes panoramas de riesgo rendimiento.

A continuación se presentan las siguientes conclusiones en dos partes: 1) a través de métricas clásicas de desempeño de portafolio y 2) en términos de AG. En una primera instancia probamos el sector de materiales derivado del crecimiento de las materias primas desde un contexto internacional discutido en Mascareñas y Alvarez-Pallete (2005), donde mencionan que China ha aumentado fuertemente la demanda por materias primas en Latinoamérica durante el 2004, lo cual ha hecho que aumenten los precios de éstas y con ello el desempeño de mercado de este sector como fue comprobado por diferentes autores (Gruian, 2011; Comisión Europea, 2009).

Otro sector con desempeño alto, según diferentes métricas de desempeño de portafolio, fue el de servicios financieros. Esto se puede deber a que México estaba en un excelente momento económico durante el periodo 2008-2012; además Estados Unidos anunció baja de tasas que todos los fondos buscaron donde invertir; finalmente el sector financiero en México era el más rentable porque la banca estaba bien capitalizada. Diversos factores explican la globalización en el sector financiero según BBVA (2007): desde capitalización de instituciones en economías emergentes; avances en tecnología, comunicaciones y gestión de riesgos; así como la búsqueda de nuevos mercados por desarrollar y que ofrezcan tasas de retorno atractivas. Así, no es accidental que el valor de las fusiones y adquisiciones en el sector financiero, tan sólo en economías emergentes, sumara en 2005 más de 20 mil millones de dólares; en 1995, una década atrás, la cifra no llegó siquiera a los 2 mil millones.

Por lo que respecta a telecomunicaciones es el sector con peor desempeño en las tres métricas estudiadas. A pesar que en el periodo 2000-2004 llegó a 73\% la inversión extranjera, incrementó 11 puntos porcentuales en diversos sectores como 
telecomunicaciones; comercio; restaurantes y hoteles; $y$, muy especialmente, intermediación financiera (BBVA, 2007).

Por otra parte, el sector industrial está compuesto por empresas del sector de construcción, las cuales han tenido un desempeño muy pobre. El año 2013 ha sido un año complejo para la industria de la construcción y el mercado de vivienda (Banxico, 2009). La desaceleración en el ritmo de actividad ha sido importante, pues el PIB pasó de registrar tasas de crecimiento de $4.3 \%$ en promedio entre 2010 y 2012 , a $1 \%$ en el primer semestre del año actual, en términos reales. Lo correspondiente a la parte residencial se ha ajustado a la baja.

Finalmente, obtuvimos resultados muy similares y más contundentes con la optimización de una función objetivo mediante AG; nuevamente el sector de servicios financieros es el que obtiene mayor ponderación.

\section{Referencias}

Arnone, S., A. Loraschi y A. Tettamanzi (1993). A genetic approach to portfolio Selection. Neural Network World 6: 597-604.

Artikis, P. y G. Nifora (2011). The Industry Effect On The Relationship Between Leverage and Returns. Eurasian Business Review 1 (2): 125-145.

Asoh, H. y H. Mühlenbein (1994). On the mean convergence time of evolutionary algorithms without selection and mutation. En Y. Davidor, P. Schwefel and R. Manner. Parallel Problem Solving from Nature PPSN III. Berlin: Springer-Verlag: 88-97.

Baird, P., P. Geylani y J. Roberts (2012). Corporate Social and Financial Performance Re-Examined: Industry Effects in a Linear Mixed Model Analysis. Journal of Business Ethics 109 (3): 367-388.

Baležentis, A., T. Baležentis y A. Misiūnas (2012). An Integrated Assessment Of Lithuanian Economic Sectors Based On Financial Ratios And Fuzzy Mcdm Methods. Technological and Economic Development of Economy 18 (1): 34-53. 
Banxico (2008). Informe anual 2007. México: Banco de México.

(2009). Informe anual 2008. México: Banco de México.

(2013). Informe anual 2012. México: Banco de México.

BBVA (2007). Situación sectorial regional. México: BBVA Research. (2013). Situación Inmobiliaria: México. México: BBVA Research.

Chamberlain, G. (1983). A characterization of the distributions that imply meanvariance utility functions. Journal of Economic Theory 29: 185-201.

Chang, T.J., S.C. Yang y K.J. Chang (2009). Portfolio optimization problems in different risk measures using genetic algorithm. Expert Systems with Applications 36: 10529-10537.

Comisión Europea (2009). Sectoral Growth Drivers and Competitiveness in the European Union. Luxembourg: Office for Official Publications of the European Communities.

Cowles, A. (1933). Can stock market forecasters forecast? Econométrica 1 (3): 206-214.

Deb K. (1997). Limitations of evolutionary computation methods. En T. Bäck, D.B. Fogel y Z. Michalewicz (eds.). Handbook of Evolutionary Computation. Oxford: IOP Publishing and Oxford University Press: B2.9.

El Milhoub, T., A.A. Hopgood, L. Nolle y A. Battersby (2006). Hybrid Genetic Algorithms: A Review. Enginnering Letter 13 (2): 124-137

Fabozzi, F., F. Gupta y H. Markowitz (2002). The legacy of modern portfolio theory. Journal of Investing 11 (3): 7-22.

Glover, F. y M. Laguna (1997). Tabu search. Boston: Kluwer Academic Publishers. 
, M. Laguna y R. Martí (2000). Fundamentals of scatter search and path relinking. Control and Cybernetics 29 (3): 653-684.

Gruian, C. (2011). The Financial Performance of European Companies: A Comparative Approach. Annals of the University of Oradea, Economic Science Series 20 (1): 193-200.

Holland, J.H. (1975). Adaptation in natural and artificial systems: An introductory analysis with applications to biology, control and artificial intelligence. University of Michigan Press.

Jensen, C.M. (1968). The performance of mutual funds in the period 1945-1964. Journal of Finance 23 (2): 389-415.

(1969). Risk, the pricing of capital assets, and the evaluation of investment portfolios. Journal of Business 42 (2): 167-247.

Krishnasamy, V. (2011). Genetic Algorithm for Solving Optimal Power Flow Problem with UPFC. International Journal of Software Engineering and its Applications 5 (1): 39-49.

Kyong, J.O., Y.K. Tae y M. Sungky (2005). Using genetic algorithm to support portfolio optimization for index fund management. Expert Systems with Applications 28: 371-379.

Lin, C. C. y Y. T. Liu (2008). Genetic algorithms for portfolio selection problems with minimum transaction lots. European Journal of Operational Research 185: 393-404.

Mahmud, H. M. (2011). Sector-Wise Stock Return Analysis: An Evidence from Dhaka Stock Exchange in Bangladesh. International Journal of Business and Management 6 (6): 276-285.

Mangram, M. (2013). A Simplified Perspective Of The Markowitz Portfolio Theory. Global Journal of Business Research 7 (1): 59-70.

Markowitz, H.M. (1952). Portfolio selection. The Journal of Finance 7 (1): 77-91. 
Martí, R. y M. Laguna (2002). Búsqueda dispersa. 1er Congreso español sobre algoritmos evolutivos y bioinspirados. Mérida (España): 302-307.

Mascareñas J. y Álvarez-Pallete, J. (2006). China - Latinoamérica: ¿Competencia o Cooperación? En Beneyto, J. M. y Argerey, P. (Coords). Europa y América Latina. El otro diálogo trasatlántico. Madrid: Biblioteca Nueva: 295-318.

Merton (1972). An analytical derivation of the efficient portfolio frontier. The Journal of Financial and Quantitative Analysis 7 (4): 1851-1872.

Michalewicz, Z. (1996). Genetic Algorithms + data structures=evolution programs. Berlín: Springer-Verlag.

Ocampo, J. (2011). ¿Cómo fue el desempeño de América Latina durante la crisis financiera actual? Ensayos Económicos 61-62: 7-33

Owen, J. y R. Rabinovitch (1983). On the class of elliptical distributions and their applications to the theory of portfolio choice. Journal of Finance 38: 745752.

Pereira, R. (2000). Genetic Algorithm Optimization for Finance and Investments. MPRA Paper, 8610, University Library of Munich, Germany.

Roudier, F. (2007). Portfolio Optimization and Genetic Algorithms. Master's Thesis Department of Management, Technology and Economics. Chair of Entrepreneurial Risks. Zurich, May 17, 2007.

Saavedra, M. L. y M. J. Saavedra (2012). El valor económico agregado y su relación con el valor agregado de mercado, la utilidad por acción y el rendimiento de los activos, en México: 2001-2008. Revue Sciences de Gestión 90: $19-40$.

Sefiane, S. y M. Benbouziane (2012). Portfolio Selection Using Genetic Algorithm. Journal of Applied Finance \& Banking 2 (4): 143-154.

Scholz, H., 2007. Refinements to the Sharpe ratio: comparing alternatives for bear Markets. Journal of Asset Management 7 (5): 347-357. 
Sharpe, W. (1966). Mutual fund performance. Journal of Business 39: 119-138.

Talbi (2002). A Taxonomy of hybrid metaheuristics. Journal of Heuristics 8 (5): 541564.

Tarek, A. El-Milhoub, A.A. Hopgood, L. Nolle y A. Battersby (2006). Hybrid Genetic Algorithms: A Review. Engineering Letters 13 (3): 124-137.

Vallée, T. y M. Yildizoglu (2001). Présentation des algorithmes génétiques et leurs applications en économie. Revue d'économie politique 4 (2): 1-23.

Venugopal, M., S. Subramanian y U. Rao (2004). Usefulness of Genetic Algorithm Model For Dynamic Portfolio Selection. Journal of Financial Management and Analysis 17 (1): 45-53.

Yu, L., S. Wang y K. Lai (2009). Multi-Attribute Portfolio Selection with Genetic Optimization Algorithms. INFOR 47 (1): 23-30.

S. Wang y F. Wen (2012). Genetic Algorithm-Based Multi-Criteria Project Portfolio Selection. Annals of Operation Research 197 (1): 71-86. 